\title{
Identifying the Roles of Medical Providers when Addressing Barriers to HPV Vaccination Rates in Rural NE Clinics
}

\author{
Abby Laudi ${ }^{1 *}$, Meera Varman ${ }^{2}$ and Amelia Simmons ${ }^{3}$ \\ ${ }^{1}$ Creighton University School of Medicine, Omaha, NE USA. \\ ${ }^{2}$ Pedatric Infectious Disease, Creighton University Omaha, NE USA. \\ ${ }^{3}$ Pedatric Infectious Disease Creighton University School of Medicine, Omaha, NE USA.
}

*Corresponding author: Abbay Laudi, Creighton University School of Medicine, Omaha, NE USA.

Received date: August 20, 2021; Accepted date: November 18, 2021; Published date: January 03, 2021

Citation: Laudi A., Varman M., Simmons A. (2022) Identifying the Roles of Medical Providers when Addressing Barriers to HPV Vaccination Rates in Rural NE Clinics. J. Obstetrics Gynecology and Reproductive Sciences 6(1) DOI:10.31579/2578-8965/093

Copyright: (C) 2022, Abbay Laudi, This is an open access article distributed under the Creative Commons Attribution License, which permits unrestricted use, distribution, and reproduction in any medium, provided the original work is properly cited.

\begin{abstract}
Background: Although many interventions to address vaccine hesitancy until now have operated on the presumption that misperceptions are due to a lack of knowledge about HPV and the vaccine, this may not always be the reason behind vaccine hesitancy. Nyhan et. al found [1] that correcting myths about vaccines- such as autism links or vaccine side effects- do not increase vaccine rates among adolescents. Medical providers play a crucial role on influencing parents' decision to vaccinate. In a study exploring how vaccination coverage among children 19-35 months old is associated with health care providers' influence on the parents' decision to vaccinate, parents who report their providers as being influential in the study are twice as likely to respond that vaccines are safe for children [2]. In the 2016 Clinical Report on Countering Vaccine Hesitancy by the American Academy of Pediatrics, motivational interviewing is listed as a potential communication technique that may be useful as pediatricians discuss vaccines with vaccine-hesitant parents.
\end{abstract}

Research Question: The research attempting to find the best approaches to reverse the increasing rates of unvaccinated minors is limited and inconclusive. This project addressed the impact of medical providers' attitudes of HPV vaccination on their early adolescent patient populations.

Methods: Our cohort prospective study first examined medical providers' baseline attitudes and approaches of HPV vaccination in privately insured clinics in rural areas of Nebraska. A survey was sent to eleven Phase III patient centered medical home (PCMH) NE clinics. The survey assessed medical staffs' attitudes and approaches to HPV vaccination, particularly among specific patient age groups. In addition to each clinic's collective survey responses, baseline HPV vaccination data was collected at eleven Phase $3 \mathrm{PCMH}$ rural clinics in Nebraska for pediatric patients 11-15-years-old. The follow-up intervention implemented educational interventions in the clinics to increase HPV vaccination rates for pediatric patients 9-15-years-old. Our educational outreach program at the selected 10-13 clinics will serve as these rural clinics' first efforts to selectively work toward improving HPV vaccination rates.

Results: America's Health Rankings (2017) found 42.4\% of adolescents living in rural areas compared to 52.4\% in urban areas are up-to-date on their HPV immunizations. The eleven rural clinics selected for the study show only a $0.9 \%$ completion of the vaccine series for 9-11-year-old patients $(n=855)$, and 25.0\% completion of the series for 12-15-year-old patients $(n=1268)$ as of 2019. This implies a pressing health disparity that needs addressing in rural Nebraskan communities. $92.6 \%$ of all respondents chose the 12-15 age range as the patient population the clinics would typically ask about the vaccine versus $59.6 \%$ who chose the 9-11 age range. The most chosen reason for not mentioning the HPV vaccine is "parents previously voiced vaccine hesitancy" (33.3\%) followed by "not enough clinic time" $(22.2 \%)$. The most popular reason contributing to parental hesitancy is "they have concerns the vaccine is not safe for their child" (70.4\%). The greatest benefit of the HPV vaccine was listed as "prevention from multiple forms of cancer" (33.3\%) and the greatest drawbacks were both "multiple dose series completion" (40.7\%) and "difficulty in convincing parents to vaccinate minors" (40.7\%). The 9-11 age range was chosen as the most difficult age group to vaccinate $(33.3 \%)$. The most difficult scenarios when addressing HPV vaccination concerns were "lack of vaccine education" (55.6\%), "religious reasons against the vaccine (44.4\%), and "language/cultural barriers" (37\%). 
Qualitative results were also analyzed separately and focused on each individual clinic's strengths and weaknesses regarding vaccination encouragement.

Discussion: The baseline patient data show that clinics selected for the study exhibit a large disparity of HPV vaccination rates among a vulnerable age group. Survey responses show both a clinical observation regarding parents' low-level education levels about the HPV vaccine as well as a lack of comfort engaging in open dialogue between patients and healthcare personnel. Focusing on these two variables alone could help increase rates of vaccination significantly. Survey results ultimately illustrate the urgent need for empirically-supported educational resources that will enhance communication- both within individual clinics among staff as well as between medical staff and patients' families- to sustainably increase HPV vaccination rates across rural clinics.

Keywords: HPV; vaccination; vaccine hesitation; public health; adolescent health; sexual health; primary care; vaccine awareness; family medicine; preventive medicine

\section{Summary Box}

\section{"What is already known on this subject?"}

Although many interventions to address vaccine hesitancy until now have operated on the presumption that misperceptions are due to a lack of knowledge about HPV and the vaccine, this may not always be the reason behind vaccine hesitancy. Nyhan et al [3] found that correcting myths about vaccines- such as autism links or vaccine side effects- do not increase vaccine rates among adolescents. Medical providers play a crucial role on influencing parents' decision to vaccinate. In a study exploring how vaccination coverage among children 19-35 months old is associated with health care providers' influence on the parents' decision to vaccinate, parents who report their providers as being influential in the study are twice as likely to respond that vaccines are safe for children [4]. In the 2016 Clinical Report on Countering Vaccine Hesitancy by the American Academy of Pediatrics, motivational interviewing is listed as a potential communication technique that may be useful as pediatricians discuss vaccines with vaccine-hesitant parents.

\section{"What does this study add?"}

There is a growing body of literature regarding the existence of vaccine hesitation in the United States and theories as to why rates of vaccine rejection are substantially increasing. However, the research attempting to find the best approaches to reverse the increasing rates of unvaccinated minors is limited and inconclusive. This project will address the impact of medical providers' attitudes of HPV vaccination on their early adolescent patient populations. Also, given the scarce research of rural health care and the need for vaccine rate improvement in rural areas, rural health access will be an area of focus in this study. Because this is a research question focusing on quality improvement, minimal IRB review is necessary to conduct research in these rural areas.

\section{Literature Review}

\section{Disease Burden}

Human papillomavirus (HPV) is a group of 200 related viruses. It is the most common sexually transmitted infection in the United States [5]. Most HPV cases are asymptomatic and result in no clinical disease. However, the main burden of HPV-related clinical disease is due to cervical cancer. Approximately $70 \%$ of cervical cancers and precancerous cervical lesions are associated with HPV types 16 and 18 [6]. Roughly $\$ 8$ billion is spent annually on management of HPV infections; primarily for abnormal cervical cytology and treatment of cervical neoplasia. The economic burden of HPV exceeds other sexually transmitted infection except human immunodeficiency virus [8].

\section{Disease Transmission}

HPV is transmitted by direct contact with an infected individual. Transmission frequently occurs from sexual intercourse but may result from non-penetrating sexual activity. Risk factors for HPV infection are primarily related to sexual behavior which includes lifetime and recent sex partners [9]. Results of epidemiologic studies are less consistent for other risk factors including young age at sexual initiation, number of sexual partners, genetic factors, smoking and lack of circumcision of male partner. Condom use is postulated to reduce transmission of HPV and other STDs but fails to completely prevent risks for transmission [10].

\section{Vaccination}

Quadrivalent HPV (qHPV) vaccine was approved by the FDA in June 2006[11]. The vaccine commonly known as Gardasil is approved for females and males 9 through 26 years of age and contains types 16 and 18 (high risk) and types 6 and 11 (low risk) [12].

The nine-valent $(9 \mathrm{v})$ Gardasil vaccine is approved for use in females and males from ages 9-26 years and includes HPV strains 16, 18, 31, 33, 45, 52, and 58, and low risk strains 6 and 11[13]. These are the major strains of HPV known to cause pathology in humans - particularly lesions that may lead to anal, cervical, vaginal, and penile or oropharyngeal cancers [14]. Earlier administration of the vaccine decreases likelihood to prior HPV exposure. CDC has updated recommendations for immunocompetent children as early as 11 up to 14 years old to receive two doses of the same type of HPV vaccine at a 6-12-month interval [15]. This time interval of dosing enhances vaccine effectiveness. A three-shot series is required for individuals who start the vaccine series 15 years of age or older [16]. Three dose series is recommended at 1-2 months and six-month intervals from the initial dose [17].

\section{Vaccine Compliance}

Despite the national vaccine recommendations, data from 2017 depicts only $50 \%$ of adolescent females in the United States have received two or three doses of the HPV vaccine by 13-15-years-old, and $42 \%$ of adolescent males [18]. In Nebraska, the rates for both sexes are higher than the national average: adolescent females report $61.4 \%$ vaccination rate and $55.2 \%$ for adolescent males [19].

Additional study findings [20] show a decrease in compliance among children younger than 13-years-old, although this is within the CDC recommended age range to receive the vaccine. A decreased compliance in the 11-13-year-old patients may be associated with parental beliefs or education about HPV. In a qualitative study that enrolled the mothers of 8-14-year-old girls, most mothers sampled in the study [21] showed enthusiasm about vaccination; however, some mothers express concern that HPV vaccination could lead to an increase in risky sexual behavior and therefore felt uncomfortable discussing the vaccine with their younger girls. HPV vaccination has not been shown to increase sexual behavior among young females [22].

\section{Physicians' Roles in Vaccination}

Although many interventions to address vaccine hesitancy until now have operated on the presumption that misperceptions are due to a lack of 
knowledge about HPV and the vaccine, this may not always be the reason behind vaccine hesitancy. In some cases, simply providing information often does not lead to people changing their views and may even create a dynamic in which a patient or parent is actually less receptive to information a provider may impart. One study [23]. Found that correcting myths about vaccines- such as autism links or vaccine side effects- do not increase vaccine rates among adolescents. Medical providers play a crucial role on influencing parents' decision to vaccinate. Another study exploring how vaccination of children 19-35 months old is associated with health care providers' influence on the parents' decision to vaccinate, parents who report their providers as being influential in the study are twice as likely to respond that vaccines are safe for children [24].

The 11-12-year-old age range is a particularly vulnerable population to the beliefs of their parents, since they typically lack sufficient knowledge about preventive medical efforts such as vaccination as well as their importance. Missed clinical opportunities to vaccinate by providers is a major barrier for improving HPV vaccination. Vadaparampil et. al [25] found that physicians report a significantly lower recommendation rate to their early adolescent patients compared with their middle and late adolescent/young adult patients. To mitigate this link, Gilkey et. al [26] found that physicians prioritizing cancer prevention is the best approach instead of addressing mode of transmission for increasing guidelineconsistent HPV vaccination.

In addition to pro-vaccine statements such as cancer prevention, the use of motivational interviewing is a growing area of research due to its effective approach to encourage families towards vaccination. As the parent starts to willingly engage in conversation, the provider can strategically pivot the conversation from the parent's concerns to disease process at hand, because there is some evidence that focusing on the disease rather than the barrier is more likely to improve intention to vaccinate [27].

\section{Research Question}

There is a growing body of literature regarding the existence of vaccine hesitation in the United States and theories as to why rates of vaccine rejection are substantially increasing. However, the research attempting to find the best approaches to reverse the increasing rates of unvaccinated minors is limited and inconclusive. This project will address the impact of medical providers' attitudes of HPV vaccination on their early adolescent patient populations. Also, given the scarce research of rural health care and the need for vaccine rate improvement in rural areas, rural health access will be an area of focus in this study. Because this is a research question focusing on quality improvement, minimal IRB review is necessary to conduct research in these rural areas.

\section{Methods}

\section{Study Design}

The study examined medical providers' baseline attitudes about the HPV vaccine in 11 Phase III patient centered medical home (PCMH) clinics in Nebraska. An online survey platform was used to gather responses. The survey assessed for attitudes and approaches to HPV vaccination among specific patient age groups. It included one-choice answer questions, multiple-choice answer questions, and two supplemental fill-in-the-blank questions to gain further clarification. In addition to each clinic's collective survey responses, baseline HPV vaccination data will be collected from the clinics regarding 11-15-year-old patients.

\section{Patient Involvement}

No patients were individually recruited for this study. We instead used aggregate data from clinics involved in the study that included how many 11-15 y/o patients are seen at each clinic and how many of them have started or completed the HPV vaccine series.

\section{Results and Analysis}

\section{Baseline Clinic Data}

Prior to data collection, anonymous patient demographic information based on age range $(9-11,12-15)$ was collected and aggregated among the eleven clinics we surveyed $(n=2123)$. Between ages 9-11-years-old $(\mathrm{n}=855), 98.1 \%$ have received zero HPV vaccine doses; $0.9 \%$ have received one dose, and $0.9 \%$ have received two doses. Between ages 12 15 -years-old $(\mathrm{n}=1268), 54.4 \%$ have received zero HPV vaccine doses; $20.5 \%$ have received one dose, and $25.0 \%$ have received two doses. questions, and two supplemental fill-in-the-blank questions to gain further clarification. In addition to each clinic's collective survey responses, baseline HPV vaccination data will be collected from the clinics regarding 11-15-year-old patients.

\section{Patient Involvement}

No patients were individually recruited for this study. We instead used aggregate data from clinics involved in the study that included how many 11-15 y/o patients are seen at each clinic and how many of them have started or completed the HPV vaccine series.

\section{Baseline Clinic Data}

Prior to data collection, anonymous patient demographic information based on age range $(9-11,12-15)$ was collected and aggregated among the eleven clinics we surveyed $(n=2123)$. Between ages 9-11-years-old $(n=855), 98.1 \%$ have received zero HPV vaccine doses; $0.9 \%$ have received one dose, and $0.9 \%$ have received two doses. Between ages 1215 -years-old $(\mathrm{n}=1268), 54.4 \%$ have received zero HPV vaccine doses; $20.5 \%$ have received one dose, and $25.0 \%$ have received two doses.

\section{Study Sample}

Personnel of various specialties representing eleven Phase III PCMH clinics participated in the baseline survey. Clinic managers from each clinic were sent the survey via email to disseminate to each clinic's personnel. Two clinics do not have personnel who elected to take the survey, so these clinics are not included in the final sample results and analysis. $3.7 \%$ of survey respondents are male, and $92.6 \%$ are female. $88.9 \%$ of the participants are White. $18.5 \%$ are medical practitioners (MD), $18.5 \%$ are nurse practitioners (NP), $37 \%$ licensed practical nurses (LPN), 22.2\% registered nurses (RN), and 3.7\% medical assistants. Ages of respondents range from 20-70-years-old, with $30 \%$ participants in the $30-39$-year-old range, $18.5 \%$ in the $40-49$ range, and $37 \%$ in the $50-59$ range. Table 1 shows the final study sample demographics $(n=27)$.

\section{Quantitative Results}

Table: 2 Survey 


\begin{tabular}{|c|c|}
\hline $\begin{array}{l}\text { 1. What age range best represents the patient } \\
\text { population that you would typically ask } \\
\text { regarding the HPV vaccine? Please select ALL } \\
\text { ranges that apply. }\end{array}$ & $\begin{array}{ll}\text { A. } & 5-8 \mathrm{y} / \mathrm{o} \\
\text { B. } & 9-11 \mathrm{y} / \mathrm{o} \\
\text { C. } & 12-15 \mathrm{y} / \mathrm{o} \\
\text { D. } & 16-18 \mathrm{y} / \mathrm{o} \\
\text { E. } & 19-21 \mathrm{y} / \mathrm{o} \\
\text { F. } & 22-26 \mathrm{y} / \mathrm{o} \\
\text { G. } & 27+\mathrm{y} / \mathrm{o}\end{array}$ \\
\hline $\begin{array}{l}\text { 2. In your opinion, what age group is ideal to } \\
\text { begin series for HPV vaccines? Please select } \\
\text { ALL ranges that apply. }\end{array}$ & $\begin{array}{l}\text { A. } 5-8 \text { y/o } \\
\text { B. } 9-11 \mathrm{y} / \mathrm{o} \\
\text { C. } 12-15 \mathrm{y} / \mathrm{o} \\
\text { D. } 16-18 \mathrm{y} / \mathrm{o} \\
\text { E. } 19-21 \mathrm{y} / \mathrm{o} \\
\text { F. } 22-26 \mathrm{y} / \mathrm{o} \\
\text { G. } 27+\mathrm{y} / \mathrm{o}\end{array}$ \\
\hline $\begin{array}{l}\text { 3. List the reason(s) you do not mention the HPV } \\
\text { vaccine during an appointment with an } \\
\text { unvaccinated minor who is in the CDC } \\
\text { recommended age range. Please choose ALL } \\
\text { that apply. }\end{array}$ & $\begin{array}{l}\text { A. Not enough clinic time } \\
\text { B. Not as high a priority } \\
\text { C. Feel uncomfortable discussing with patient/parents } \\
\text { D. Parents previously voiced vaccine hesitancy } \\
\text { E. They forget to mention it } \\
\text { F. Other (please list) }\end{array}$ \\
\hline $\begin{array}{l}\text { 4. What reasons contribute to your parents' } \\
\text { hesitancy to vaccine for HPV? Please choose } \\
\text { ALL that apply. }\end{array}$ & $\begin{array}{l}\text { A. They did not know about the HPV vaccine. } \\
\text { B. They have concerns that the vaccine is not safe for their child. } \\
\text { C. The child is not comfortable receiving injections. } \\
\text { D. Their child is too young to receive the vaccine at this time. } \\
\text { E. Their child does not need the vaccine because child is male. } \\
\text { F. Their child does not need the vaccine because their child is not } \\
\text { G. Concerns regarding the child's other health issues. } \\
\text { H. They have cultural, religious, and/or personal beliefs that discourage } \\
\text { I. or prevent their child to receive vaccinations. }\end{array}$ \\
\hline $\begin{array}{l}\text { 5. In your opinion, what is the greatest benefit of } \\
\text { the HPV vaccine? Please choose one. }\end{array}$ & $\begin{array}{l}\text { A. Lifelong protection against HPV. } \\
\text { B. Prevention from multiple forms of cancer } \\
\text { C. Little to no side effects } \\
\text { D. Financial incentives received with increased vaccination rates } \\
\text { E. Increased public understanding about the severity of HPV } \\
\text { F. Willingness of parents to be educated about HPV and the vaccine } \\
\text { G. Other (please list) }\end{array}$ \\
\hline $\begin{array}{l}\text { 6. In your opinion, what is the greatest drawback } \\
\text { of the HPV vaccine? Please choose one. }\end{array}$ & $\begin{array}{l}\text { A. A. Inconvenience by adding to work load } \\
\text { B. Difficulty in convincing parents to vaccinate minors } \\
\text { C. Promotes earlier sexual activity in minors } \\
\text { D. Contradicts my own religious/personal beliefs as a provider } \\
\text { E. Multiple dose series completion } \\
\text { F. Lacks sufficient data to prove efficacy } \\
\text { G. Other (please list) }\end{array}$ \\
\hline $\begin{array}{l}\text { 7. What is the most difficult age group to } \\
\text { vaccinate against HPV? Please choose one. }\end{array}$ & $\begin{array}{ll}\text { A. } & 5-8 \mathrm{y} / \mathrm{o} \\
\text { B. } & 9-11 \mathrm{y} / \mathrm{o} \\
\text { C. } & 12-15 \mathrm{y} / \mathrm{o} \\
\text { D. } & 16-18 \mathrm{y} / \mathrm{o} \\
\text { E. } & 19-21 \mathrm{y} / \mathrm{o} \\
\text { F. } & 22-26 \mathrm{y} / \mathrm{o} \\
\text { G. } & 27+\mathrm{y} / \mathrm{o}\end{array}$ \\
\hline $\begin{array}{l}\text { 8. In your opinion, who is primarily responsible } \\
\text { for ensuring vaccination of the pediatric patients } \\
\text { who come to your clinic? Please choose one. }\end{array}$ & $\begin{array}{l}\text { A. Medical provider } \\
\text { B. Medical provider }+ \text { physician assistant/nurse practitioner } \\
\text { C. Medical provider }+ \text { physician assistant/nurse practitioner }+ \text { nurses } \\
\text { D. Medical provider }+ \text { physician assistant/nurse practitioner }+ \text { nurses }+ \\
\text { front desk staff } \\
\text { E. Parents of the child } \\
\text { F. Other }\end{array}$ \\
\hline
\end{tabular}


9. Please explain your previous response in a brief sentence or two.

10. Based on your experience, which of the following scenarios is most difficult to address HPV vaccination concerns? Please choose ALL that apply.

11. Select ALL the following protocols your clinic currently uses to increase HPV vaccination rates:
A. Religious reasons against the vaccine
B. Gender of the child is female
C. Language/cultural barriers regarding the vaccine
D. Reasons related to sex/sexual activity of their child
E. Gender of the child is male
F. Perception that the child Is too young to receive the vaccine
G. Lack of education regarding the vaccine (safety, efficacy, etc.)
H. Other (please explain)
A. Daily huddle
B. Reminder recalls sent to parents of 9-19-year-old minors due for the vaccine
C. Front staff communication with providers is required to assess vaccination status of patients coming in sometime within the week
D. Educational materials in the waiting area
E. Educational brochures given to parents of 9-19-year-old minors due for the vaccine
F. Reports given to providers about vaccination rates at your clinic
G. Vaccination rates are shared with the entire clinic staff
H. Special protocol for vaccine-hesitant families to encourage vaccination
I. Follow up appointments are made the same day after the dose

\section{What allows your clinic to do well with targeting the CDC recommended age range for vaccination? Please explain briefly below. \\ 13. What could your clinic improve upon in order to increase HPV vaccination rates at your clinic? Please explain briefly below.}

Table 1 shows each survey question and response options, and specifies if the question is a multiple-choice, single-choice, or fill-in-the-blank response.

\section{Qualitative Results}

Question 9 asks for a brief statement defending the respondent's answer to Question 8: "In your opinion, who is primarily responsible for ensuring vaccination of the pediatric patients who come to your clinic?" For the majority who thought the entire team is responsible for vaccination (63\%), responses included: it is the whole team to ensure patients are educated; currently it is just the PA or MD that orders and recommends it, we need to empower and challenge our nurses and other clinical staff to encourage the HPV vaccine. For those who answered Question 9 with the medical provider as primarily responsible (14.8\%), responses included: providers need to inform the parent/patient of the benefits and risks of vaccination so patient/parent are aware.

Questions 12 and 13 ask respondents to explain their own opinions regarding what their respective clinics do well when targeting the CDC age range for vaccination, and where there is room for improvement. Table 3 shows themes for these questions based on the recorded responses.

\begin{tabular}{|c|c|c|c|c|c|}
\hline Question & Theme & & & & \\
\hline $\begin{array}{l}\text { 12. What allows your clinic to } \\
\text { do well with targeting the CDC } \\
\text { recommended age range for } \\
\text { vaccination? Please explain } \\
\text { briefly below. }\end{array}$ & $\begin{array}{l}\text { Medical technology to } \\
\text { flag who is due for a } \\
\text { vaccine prior to } \\
\text { appointments }\end{array}$ & \begin{tabular}{|l|} 
Up-to-date \\
vaccine \\
schedule in \\
patients' \\
immunization \\
records
\end{tabular} & $\begin{array}{llr}\text { Discussion } & \text { of vaccines } \\
\text { becomes } & \text { an option } \\
\text { during } & \text { well-child } \\
\text { checkups } & & \end{array}$ & $\begin{array}{l}\text { Clinics receive } \\
\text { educational information } \\
\text { that personnel can } \\
\text { utilize to teach patients } \\
\text { and parents }\end{array}$ & \\
\hline $\begin{array}{l}\text { 13. What could your clinic } \\
\text { improve upon in order to } \\
\text { increase HPV vaccination } \\
\text { rates at your clinic? Please } \\
\text { explain briefly below. }\end{array}$ & $\begin{array}{l}\text { Improved } \\
\text { communication } \\
\text { between all clinic staff } \\
\text { (daily huddles, etc.) }\end{array}$ & $\begin{array}{l}\text { Increase } \\
\text { patient } \\
\text { education } \\
\text { resources }\end{array}$ & $\begin{array}{l}\text { Provide high-yield } \\
\text { informational handouts } \\
\text { prior to patients seeing } \\
\text { the provider (lobby, } \\
\text { waiting area, etc.) }\end{array}$ & $\begin{array}{l}\text { Improve the follow-up } \\
\text { appointments protocol }\end{array}$ & $\begin{array}{l}\text { Ability to give } \\
\text { vaccines to all } \\
\text { eligible children, } \\
\text { regardless of } \\
\text { insurance status }\end{array}$ \\
\hline
\end{tabular}

Table 2: Qualitative Themes for Open-Ended Survey Questions

\section{Discussion}

$42.4 \%$ of adolescents living in rural areas compared to $52.4 \%$ in urban areas are up-to-date on their HPV immunizations [28]. The eleven rural clinics selected for the study show only a $0.9 \%$ completion of the vaccine 
series for 9-11-year-old patients $(n=855)$, and $25.0 \%$ completion of the series for $12-15$-year-old patients $(n=1268)$ as of 2019 . This implies a pressing health disparity that needs addressing in rural Nebraskan communities.

Survey responses illustrate a prominent lack of parental knowledge and awareness about the vaccine and its safety profile at these clinics. Responses also show parental unawareness regarding the benefits of the vaccine that vastly outweigh the potential side effects of vaccine administration. Thus, parental hesitation could be a valid reason contributing to the low rates of HPV vaccination among 9-11-year-olds reported in these clinics. However, these survey responses also bring to light the need for clinics to target the parents of 9-11-year-old patients with open dialogue regarding HPV vaccine education.

The survey responses help explain missed vaccination opportunities among the 9-11 patient population; if clinic personnel are reporting they are much less likely to mention the HPV vaccine to 9-11-year-olds over 12-15-year-olds, then the low vaccine rates are not solely because of parental beliefs regarding vaccine safety, etc. Previously cited literature suggests that cancer prevention is the best way to mitigate hesitation among parents of minors and convince them to vaccinate [29]. Clinic personnel agreed in their responses that the greatest benefit of the vaccine was cancer prevention closely followed by lifelong protection from HPV; personnel also thought the greatest drawback was the difficulty in convincing parents to vaccinate their children along with the multiple dose series. Thus, the survey suggests that knowledge behind the importance of the HPV vaccine is present among clinic personnel, but the education behind how to relay this knowledge in a way that will enhance communication between a provider and her patient's family is lacking.

The survey also shows that the entire clinical should be responsible for vaccinating each adolescent patient. This implies a general understanding among clinic personnel that everyone interacting with the patient has a duty to put the HPV vaccine on the parents' radar and provide any supplemental information that could help convince the patient to receive the vaccine during the visit.

Themes highlighting unique challenges to rural clinics- such as a patient's transportation access to complete the vaccine series or an inability to vaccinate all children regardless of insurance status- are implications that bring importance to more systemic issues in the healthcare system that span beyond rural Nebraska. Future studies can look at the disparities in rural areas versus their urban counterparts and how specific interventions can target one of the many unique challenges that rural clinics and their communities face.

Limitations in this study include the small sample of clinic personnel who participated in the survey. Given the small baseline number of clinic personnel per clinic, future studies could replicate this study more rural clinics to increase survey respondents. Another limitation was the lack of diversity among the survey respondents, Future studies could target different regions of the country in order to get a more diverse group of participants.

\section{Conclusion}

This research project intended to gather information regarding health care personnel's attitudes about HPV vaccination based on experiences in their respective rural clinics. The baseline patient data show that clinics selected for the study exhibit a large disparity of HPV vaccination rates among the most vulnerable age ranges allowed by the CDC to receive the vaccine. Survey responses show both a clinical observation regarding parents' low-level education levels about the HPV vaccine as well as a lack of comfort engaging in open dialogue between patients and healthcare personnel. Focusing on these two variables alone could help increase rates of vaccination significantly; by first educating clinics with relevant guidance on how to communicate with vaccine-hesitant families, clinic personnel can feel more comfortable engaging in parental education regarding the vaccine's safety and efficacy. Survey results ultimately illustrate the urgent need for empirically supported educational resources that will enhance communication- both within individual clinics among staff as well as between medical staff and patients' families- to sustainably increase HPV vaccination rates across rural clinics.

\section{Works Cited}

1. Nyhan B., Reifler J., Richey S., \& Freed G. L. (2014). Effective Messages in Vaccine Promotion: A Randomized Trial.

2. Smith, P.J., Kennedy, A.M., Wooten, K. et al. Association between health care providers' influence on parents who have concerns about vaccine safety and vaccination coverage. Pediatrics. 2006; 118: e1287-e1292

3. Nyhan B., Reifler J., Richey S., \& Freed G. L. (2014). Effective Messages in Vaccine Promotion: A Randomized Trial.

4. Smith, P.J., Kennedy, A.M., Wooten, K. et al. Association between health care providers' influence on parents who have concerns about vaccine safety and vaccination coverage. Pediatrics. 2006; 118: e1287-e1292

5. National Cancer Institute (2015). Human papillomavirus (HPV) vaccines.

6. World Health Organization (2018). Human papillomavirus (HPV) and cervical cancer..

7. National Cancer Institute (2015). Human papillomavirus (HPV) vaccines.

8. Ibid.

9. Hamborsky J., Kroger, A., \& Wolfe, S. (2015). Epidemiology and prevention of vaccine-preventable diseases.

10. Ibid.

11. Ibid.

12. Ibid.

13. Ibid.

14. National Cancer Institute (2015). Human papillomavirus (HPV) vaccines.

15. Center for Disease Control and Prevention (2019). Easy-to-read Immunization Schedule by Vaccine for Ages 7-18 Years.

16. Ibid.

17. U.S. Department of Health and Human Services. (2006). Vaccines for Infants, Children, and Teens.

18. Office of Disease Prevention and Health Promotion (2017).

19. America's Health Rankings analysis of CDC (2017). National Immunization Survey-Teen.

20. Ibid.

21. Waller J., Marlow L.A., \& Wardle J. (2006). Mothers' attitudes towards preventing cervical cancer through human papillomavirus vaccination: a qualitative study.

22. First, Lewis (2018). Does Legislation to Increase Uptake of HPV Vaccine in Teens lead to Their Increased Sexual Behavior?

23. Nyhan B., Reifler J., Richey S., \& Freed G. L. (2014). Effective Messages in Vaccine Promotion: A Randomized Trial.

24. Smith, P.J., Kennedy, A.M., Wooten, K. et al. Association between health care providers' influence on parents who have concerns about vaccine safety and vaccination coverage.

25. Vadaparampil, S. T., Kahn, J. A., Salmon, D., Lee, J., Quinn, G. P., Roetzheim, R., Giuliano, A. R. (2011). Missed clinical opportunities: Provider recommendations for HPV vaccination for 11-12 year old girls are limited.

26. Gilkey, M. B., Zhou, M., Mcree, A., Kornides, M. L., \& Bridges, J. F. (2018). Parents Views on the Best and Worst Reasons for Guideline-Consistent HPV Vaccination. 
27. Mcclure, C. C., Cataldi, J. R., \& O’Leary, S. T. (2017). Vaccine Hesitancy: Where We Are and Where We Are Going

28. America's Health Rankings analysis of CDC (2017). National Immunization Survey-Teen.

29. Gilkey, M. B., Zhou, M., Mcree, A., Kornides, M. L., \& Bridges, J. F. (2018). Parents Views on the Best and Worst Reasons for Guideline-Consistent HPV Vaccination.

\section{References}

1. Nyhan B., Reifler J., Richey S., \& Freed G. L. (2014). Effective Messages in Vaccine Promotion: A Randomized Trial. Pediatrics 133 (4) 835-842; doi: 10.1542/peds.2013-2365

2. Smith, P.J., Kennedy, A.M., Wooten, K. et al.(2006) Association between health care providers' influence on parents who have concerns about vaccine safety and vaccination coverage. Pediatrics. 118: e1287-e1292

3. National Cancer Institute (2015). Human papillomavirus (HPV) vaccines.

4. World Health Organization (2018). Human papillomavirus (HPV) and cervical cancer.

5. Hamborsky J., Kroger, A., \& Wolfe, S. (2015). Epidemiology and prevention of vaccine-preventable diseases. Communication and Education Branch of the National Center for Immunization and Respiratory Diseases: Centers for Disease Control and Prevention.

6. National Cancer Institute (2015). Human papillomavirus (HPV) vaccines.

7. Warner K. Huh, Jouyra, E.A., Giuliano, A.R., ErikIversen, O., Andrade R.P., Ault, K.A.,...Mayrand, M.H. (2017). Final efficacy, immunogenicity, and safety analyses of a nine-valent human papillomavirus vaccine in women aged $16-26$ years: a randomised, double-blind trial. The Lancet, 390 (10108), 21432159.

8. Center for Disease Control and Prevention (2019). Easy-to-read Immunization Schedule by Vaccine for Ages 7-18 Years.

9. U.S. Department of Health and Human Services. (2006). Vaccines for Infants, Children, and Teens.
10. America's Health Rankings analysis of CDC (2017). National Immunization Survey-

11. Varman, M., Sharlin, C., Fernandez, C., Vasudevan, J., \& Wichman, C. (2018). Human Papilloma Virus Vaccination Among Adolescents in a Community Clinic Before and After Intervention. Journal of Community Health, 43(3), 455-458.

12. Rahman, M., Hirth, J. M. \& Berenson, A. B. (2017). Adherence to ACIP recommendation for human papillomavirus vaccine among US adolescent girls. Journal of Community Health, 42(2), 385-389.

13. Waller J., Marlow L.A., \& Wardle J. (2006). Mothers' attitudes towards preventing cervical cancer through human papillomavirus vaccination: a qualitative study. Cancer Epidemiol Biomarkers Prev., 15(7):1257-1261.

14. First, Lewis (2018). Does Legislation to Increase Uptake of HPV Vaccine in Teens lead to Their Increased Sexual Behavior? American Academy of Pediatrics.

15. Vadaparampil, S. T., Kahn, J. A., Salmon, D., Lee, J., Quinn, G. P., Roetzheim, R., Giuliano, A. R. (2011). Missed clinical opportunities: Provider recommendations for HPV vaccination for 11-12 year old girls are limited. Vaccine, 29(47), 86348641. doi:10.1016/j.vaccine.2011.09.006

16. Gilkey, M. B., Zhou, M., Mcree, A., Kornides, M. L., \& Bridges, J. F. (2018). Parents Views on the Best and Worst Reasons for Guideline-Consistent HPV Vaccination. Cancer Epidemiology Biomarkers \& Prevention, 27(7), 762-767. doi:10.1158/1055-9965.epi-17-1067

17. Mcclure, C. C., Cataldi, J. R., \& O'Leary, S. T. (2017). Vaccine Hesitancy: Where We Are and Where We Are Going. Clinical Therapeutics, 39(8), $\quad 1550-1562$. doi:10.1016/j.clinthera.2017.07.003

18. Gilkey, M. B., Zhou, M., Mcree, A., Kornides, M. L., \& Bridges, J. F. (2018). Parents Views on the Best and Worst Reasons for Guideline-Consistent HPV Vaccination. Cancer Epidemiology Biomarkers \& Prevention, 27(7), 762-767. doi:10.1158/1055-9965.epi-17-1067

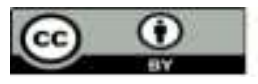

This work is licensed under Creative Commons Attribution 4.0 License

To Submit Your Article Click Here: Submit Manuscript

DOI: $10.31579 / 2578-8965 / 093$
Ready to submit your research? Choose Auctores and benefit from:

* fast, convenient online submission
* rigorous peer review by experienced research in your field
* authors retain copyrights
* unique DOI for all articles
* immediate, unrestricted online access

At Auctores, research is always in progress.

Learn more auctoresonline.org/journals/obstetrics-gynecology-andreproductive-sciences 